\title{
Assessment of occlusive disease of lower extremity arteries on the basis of anatomic region: Value of 128-slice multidetector CT angiography in comparison with digital subtraction angiography
}

\author{
Roshan Pangeni ${ }^{1}$, Ping Han ${ }^{1}$, Feng Pan ${ }^{1}$, Laxmi Pangeni Lamsal ${ }^{2}$, Zhen Zhang $^{1}$, Jie Yu $^{1}$, Rajiv Rizal ${ }^{2}$ \\ ${ }^{1}$ Department of Radiology, Union Hospital of Tongji Medical College ${ }^{2}$ Tongji Hospital of Tongji Medical \\ College
}

\section{Correspondence \\ Dr. Roshan Pangeni \\ Department of Radiology, Union \\ Hospital of Tongji Medical \\ College}

Email:

rpangeni2011@gmail.com

DOI: http://dx.doi.org/10.3126/ jcmsn.v12i4.16416

Article received: March $5^{\text {th }}$

2016

Article accepted: Sept $22^{\text {nd }} 2016$

\begin{abstract}
Background \& Objectives: The Previous studies of multidetector CT (MDCT) of the lower extremities for the detection of peripheral vascular disease showed high diagnostic accuracy but were performed with older generation systems. Our study aimed at assessing the diagnostic value of 128 MDCTA compared with that of digital subtraction angiography (DSA) in the grading of focal arterial disease of lower extremity arteries on the basis of anatomic regions. Materials \& Methods: Forty-two patients with peripheral arterial occlusive diseases underwent both MDCTA and DSA. Lower extremity arteries depicted at MDCTA and DSA were graded separately for the degree of stenosis into 3 anatomic regions and 33 segments. Grading by MDCTA and DSA was done independently. Homogeneity analysis was used between MDCTA and DSA measurements in each patient. The sensitivity, specificity, positive predictive value and negative predictive value for detection of stenotic lesions were calculated for all anatomic regions, with findings at DSA used as the reference standard. Results: No statistically significant difference $(\mathrm{P}>.05)$ between DSA and MDCTA was present in Aorto-iliac and poplitiofemoral regions while there was statistically significant difference $(\mathrm{P}<.05)$ in the infrapopliteal region. The Sensitivity, Specificity, Positive Predictive Value and Negative Predictive Value based on a reading of MDCTA were $84.3 \%, 93.8 \%, 89.4 \%$ and $90.6 \%$ for aorto-iliac $86.6 \%, 94.7 \%, 84.1 \%$ and $94.7 \%$ for poplitiofemoral and $95.7 \%, 86.1 \%, 85.6 \%$ and $95.9 \%$ for infra-popliteal region respectively.

Conclusion: MDCTA is excellent alternative in diagnosing lower extremity arterial occlusive diseases above the knee. DSA remains better on illustrating distal runoff vessels.

Key words: Digital Subtraction Angiography; Multidetector CT Angiography; Peripheral Arterial Occlusive Disease
\end{abstract}

Citation: Pangeni R, Han P, Pan F, Lamsal LP, Zhang Z, Yu J, Rizal R. Assessment of occlusive disease of lower extremity arteries on the basis of anatomic region: Value of 128 -slice multidetector CT angiography in comparison with digital subtraction angiography. JCMS Nepal. 2016;12(4):179-86.

\section{INTRODUCTION}

Lower extremity arterial diseases (also known as peripheral arterial disease or PAD) represent a significant health problem with increased morbidity and mortality. It can be sub-classified into; stenoocclusive, aneurysmal, vasculitis, traumatic vascular injuries and abnormal arterio-venous communications. In occlusive disease lumen is narrowed either in an acute or chronic manner. ${ }^{1}$

Digital sub-traction angiography (DSA) has been considered as gold standard of diagnosing peripheral vascular diseases. However, it is invasive and exposes the investigator and patient to a lot of ionizing radiation. ${ }^{2}$ DSA also is a time and cost consuming procedure. Finally, DSA results only in luminograms, and thus information about plaque constituents and vessel surroundings cannot be acquired. $^{3}$

Multi-detector computed tomography angiography (MDCT angiography) of the lower extremity has 
high accuracy for detection of steno-occlusive diseases compared with DSA. ${ }^{4}$ Its advantages over DSA includes minimal invasiveness, smaller required volume of contrast material, shorter scan time and fast data acquisition. Other advantages of MDCT angiography include three dimensional (3D) volumetric data analysis and display, visualization of mural plaque and calcium. Unlike catheter angiography, MDCTA not only depicts the vessels but also allows assessment of perfusion in adjacent organs. These advantages have led to CTA replacing DSA for diagnosis at many centers. ${ }^{5}$ However, there is a paucity of studies comparing the 128-slice MDCTA with DSA on the basis of region of blood vessel regarding grading in lower extremity.

The purpose of the present study was to evaluate the diagnostic accuracy of 128-slice MDCTA in comparison with DSA in patients with PAD depending on the region of blood vessel.

\section{MATERIALS AND METHODS \\ Subjects}

This retrospective study was performed between May 2011 and May 2013. There were a total of 42 patients who underwent both MDCTA and DSA for PAD. The mean age of the patients was 70 years, (range, 38 to 89 years), and 32 were male $(76.19 \%$ ) and 10 female $(23.81 \%)$. The local institutional review board approved this retrospective study.

\section{MDCTA}

MDCTA was performed on a Sensation 128-slice CT system (Siemens Somatom Definition AS+). Patients were in the feet first supine position on the CT table with their legs held together. The scanning range was planned to encompass the entire vascular system from the diaphragm to the level of the ankles.

For optimal intraluminal contrast enhancement, the delay time between start of contrast material administration and start of scanning was obtained for each patient individually by using a bolustracking technique (CARE-Bolus, Siemens). Subsequently, a nonionic contrast media (Iohexol injection $100 \mathrm{ml}: 35 \mathrm{~g}$ )was administered through a 20 gauge cannula that was placed into the patient's antecubital vein for a total volume of $120 \mathrm{ml}$. The contrast agent was administered with an automatic power injector (Ulrich medical missouri) at a flow rate of $4 \mathrm{ml} / \mathrm{sec}$. Data acquisition was performed craniocaudally with the following parameters: collimation $0.6 \mathrm{~mm}$, pitch 0.5 , tube voltage $100 \mathrm{KV}$, current $83 \mathrm{mAs}$, slice thickness $0.75 \mathrm{~mm}$ at an interval of $0.4 \mathrm{~mm}$. Two orthogonal curved planar reformations were created along the longitudinal axis of the aorta through both common and external iliac arteries and the common femoral artery. All data were then transferred to a workstation (Syngo Multimodality Workplace, VE36A) for post processing. The reconstructions were performed by the technologists experienced in 3D post processing and segmentation techniques. Segmentation was performed of both bony structures and vessel wall calcifications resulting in images containing the contrast enhanced vascular lumen without vessel wall calcifications and bones. Of these data sets rotating volume MIP images were generated.

\section{DSA technique}

DSA was performed using an angiography system (Siemens Artis zee). All the angiograms were performed as part of a therapeutic intervention. The arterial access site and the extent of image coverage were guided by the clinical need of the patient. One patient had only one side examined, and 41 patients had bilateral imaging of only the clinically relevant vascular segments. For bilateral studies $5 \mathrm{~F}$ pigtail catheters were placed into the abdominal aorta. Fifteen to twenty millilitres of ioversol $320 \mathrm{mg}$ iodine $/ \mathrm{ml}$ ) were injected at a rate of $10 \mathrm{ml} / \mathrm{s}$, with two frames per second for the aorto-iliac segments and one frame per second for the infra-inguinal segments. For infrapopliteal segments the amount of contrast medium injected per run could be increased to $30 \mathrm{ml}$ at the discretion of the interventionist. Unilateral studies were performed with automatic injector (Mark V ProVis) through a 4 or $5 \mathrm{~F}$ sheath or catheter of $10-20 \mathrm{ml}$ ioversol 320 . A $50 \%$ dilution was used for the poplitiofemoral segments and no dilution for the infra-popliteal segments. One frame per second was used for most injections. Anteroposterior and bilateral 350 oblique views were routinely obtained for the aortoiliac segments. For the infra-inguinal segments oblique views were obtained only when it was clinically necessary.

\section{Image and Data Analysis}

The arterial tree was divided into 32 segments for the evaluation of steno-occlusive disease as shown in the figure 1 . The arterial segments were divided into three subgroups, which included the aorto-iliac, poplitiofemoral and Infra-popliteal segments. All 32 segments were evaluated with MDCTA for all 42 patients, in a picture archiving and communication system(PACS) workstation (FUJITSU computers Siemens). For the interpretation of the CT angiograms, the axial 
Table 1 Diameter Grading System

\begin{tabular}{|cc|}
\hline Grade & Degree of Stenosis \\
\hline 1 & No Stenosis \\
\hline 2 & $<50 \%$ \\
\hline 3 & $50-75 \%$ \\
\hline 4 & $75-99 \%$ \\
\hline 5 & Occlusion \\
\hline
\end{tabular}

Table 3 Comparison of CTA with DSA Performance on Regional Basis

$\begin{array}{llll}\text { Vascular Region } & \begin{array}{l}\text { CTA } \\ \text { Median }\end{array} & \begin{array}{l}\text { DSA } \\ \text { Median }\end{array} & \begin{array}{l}\text { Palue } \\ \text { Aorto-iliac }\end{array} \\ \begin{array}{l}\text { arteries } \\ \text { Poplitiofemoral }\end{array} & 2.00 & 2.00 & 0.642 \\ \begin{array}{l}\text { arteries } \\ \begin{array}{l}\text { Infra-popliteal } \\ \text { arteries }\end{array}\end{array} & 2.00 & 2.00 & 0.461 \\ & & & 0.001 \\ \end{array}$

Table 2 Assessment of Degree of Stenosis with DSA and Multi-Detector Row CT Angiography in Aorto-iliac and Lower-Extremity Arteries

\begin{tabular}{llllllll}
\multicolumn{7}{c}{} & \multicolumn{2}{l}{ DSA Grade } & & & Total \\
& & 1 & 2 & 3 & 4 & 5 & \\
& 1 & 155 & 10 & 2 & 0 & 0 & 167 \\
$\begin{array}{l}\text { CTA } \\
\text { Grade }\end{array}$ & 2 & 33 & 219 & 8 & 1 & 0 & 261 \\
& 3 & 2 & 14 & 22 & 7 & 1 & 46 \\
& 4 & 2 & 4 & 3 & 15 & 4 & 28 \\
& Grade 5 & 0 & 0 & 4 & 8 & 145 & 157 \\
Total & & $\mathbf{1 9 2}$ & $\mathbf{2 4 7}$ & $\mathbf{3 9}$ & $\mathbf{3 1}$ & $\mathbf{1 5 0}$ & $\mathbf{6 5 9}$
\end{tabular}

images and thin MIPs were routinely evaluated. Segments that were not of diagnostic quality due to inadequate opacification, extreme calcification, or artifacts were excluded from the analysis. In every segment, the most severe lesion was graded using a five-point scale (Table 1). The DSA images were also measured with the same five-point scale after calibration. All segments that were adequately evaluated with both techniques were included in the comparative analysis. Analysis of steno occlusive disease was performed using a lesion-based approach rather than an arterial segment-based approach, as has been used in other studies (6-8).

5.Statistical analysis

The acquired data were entered in a data sheet (Excel 2010, Microsoft) and exported to a statistics program (SPSS 20 for Windows, SPSS, IL, USA). Paired non-parametric statistical analysis (homogeneity analysis) was used between MDCTA and DSA measurement, and the probability of type I error was set at 0.05. Calculations of sensitivity, specificity, positive predictive value (PPV), and negative predictive value (NPV) for the detection of the degree of all stenotic lesions were made by using DSA findings as the reference standard.

\section{RESULTS}

Forty-two patients were evaluated with both MDCTA and DSA. A total of 659 segments were covered by both CTA and DSA. On DSA images, 467 diseased segments $(70.86 \%)$ were identified: 247 with grade 2 stenosis, 39 with grade 3 stenosis, 31 with grade 4 stenosis, and 150 with occlusion. In 70 segments the degree of stenosis was overestimated at MDCTA. In 58 segments (37 of which were considered normal at DSA but were 
Table 4 Performance of 128-Detector Row CT Angiography Compared with Conventional DSA in the Detection of Arterial Stenosis

\begin{tabular}{|lllll|} 
Vascular Region & $\begin{array}{l}\text { Sensitivity } \\
(\%)\end{array}$ & Specificity(\%) & $\begin{array}{l}\text { Positive Predictive } \\
\text { Value(\%) }\end{array}$ & $\begin{array}{l}\text { Negative Predictive } \\
\text { Value(\%) }\end{array}$ \\
\hline Aorto-iliac & $84.3 \%$ & $93.8 \%$ & $89.4 \%$ & $90.6 \%$ \\
\hline Poplitiofemoral & $86.6 \%$ & $94.7 \%$ & $84.1 \%$ & $95.7 \%$ \\
\hline Infra-popliteal & $95.7 \%$ & $86.1 \%$ & $85.6 \%$ & $95.9 \%$
\end{tabular}

diagnosed as mildly stenosed at MDCTA), the degree of stenosis was overestimated at MDCTA by one grade; in 10 segments, the degree of stenosis was overestimated by two grades and in 2 segments, the degree of stenosis was overestimated by three grades. In 33 segments the degree of stenosis was underestimated at MDCTA. The degree of stenosis was underestimated by one grade in 29 segments and by two grades in 4 segments.

The comparison of CTA performance with DSA performance on regional basis by paired nonparametric statistical analysis (homogeneity analysis) was done(Table 3). For larger arteries like aorto-iliac and femoro-popliteal the $\mathrm{P}$ value is $>0.05$. While it is $<0.05$ for smaller arteries like infra-popliteal. Figure 2 and Figure 3 were true positive cases for aorto-iliac region. Figure 4 was a true positive case for poplitiofemoral region. Figure 5 was a false positive case for infra-popliteal region. Figure 5 showed the need of delayed imaging. Table 4 shows Sensitivity, Specificity, Positive Predictive Value and Negative Predictive Value.

\section{DISCUSSION}

Recent technologic advances in MDCT now allow rapid image acquisition with excellent spatial and temporal resolution. ${ }^{9}$ Advanced post-processing workstations enable rapid image manipulation and assessment. Previous studies using older generation MDCT scanners (4 and 16 slice) found adequate accuracy to detect lesions within the peripheral vasculature. $^{10}$

In current study, we extend these findings by evaluating the accuracy of the newest iteration in CT scanner, 128 MDCT. In a cohort of 42 patients with intermittent claudication, we found Sensitivity, Specificity, Positive Predictive Value and Negative Predictive Value based on a reading of multidetector row CT angiograms(Table 4).

Several previous studies have evaluated the use of peripheral CT angiography and revealed excellent correlation between MDCTA and DSA (Table 5) ${ }^{4}$ ${ }^{8,11-17}$ Met et al. ${ }^{18}$ performed a systematic review and meta-analysis for 20 studies published through 2008, that included 957 overall patients. The summary estimates of sensitivity and specificity for aorto-iliac disease provided in five studies were $96 \%$ and $98 \%$ respectively. The summary estimates of sensitivity and specificity for femoropopliteal disease provided in five studies were $97 \%$ and $94 \%$ respectively. The summary estimates of sensitivity and specificity for distal runoff in the tibial arteries provided in six studies were $95 \%$ and $91 \%$.

In the current study data was stratified on the basis of region i.e. aorto-iliac, poplitiofemoral and infra -popliteal region. Then homogeneity test was applied. There was statistically significant difference $(\mathrm{P}<.05)$ between DSA and MDCTA for infra-popliteal region while there was no statistically significant difference $(\mathrm{P}>.05)$ between DSA and MDCTA in case of aorto-iliac and poplitiofemoral region. This means MDCTA is consistent and accurate as DSA in the assessment of patients with peripheral occlusive disease of arteries and can be reliably used to grade disease severity and plan treatment in case of aorto-iliac and poplitiofemoral arteries while MDCTA is not so good as DSA in case of infra-popliteal arteries. This difference is thought to be due to close relation to bone causing more artifacts (e.g. Partial volume averaging) and small diameter of the arteries of infra-popliteal region giving rise to more error. The diameter of the proximal $(8-12$ $\mathrm{mm}$ diameter), mid (5-7 $\mathrm{mm}$ diameter), and distal (1.5-4 mm diameter) arteries are $8-12 \mathrm{~mm}, 5-$ $7 \mathrm{~mm}, 1.5-4 \mathrm{~mm}^{19}$

The sensitivity and specificity in our study bears excellent comparison as compared to other studies although it's slightly lower as compared to those studies. The scanning range in our study is larger than these studies. Our study can provide more overall idea for the clinician and interventional 

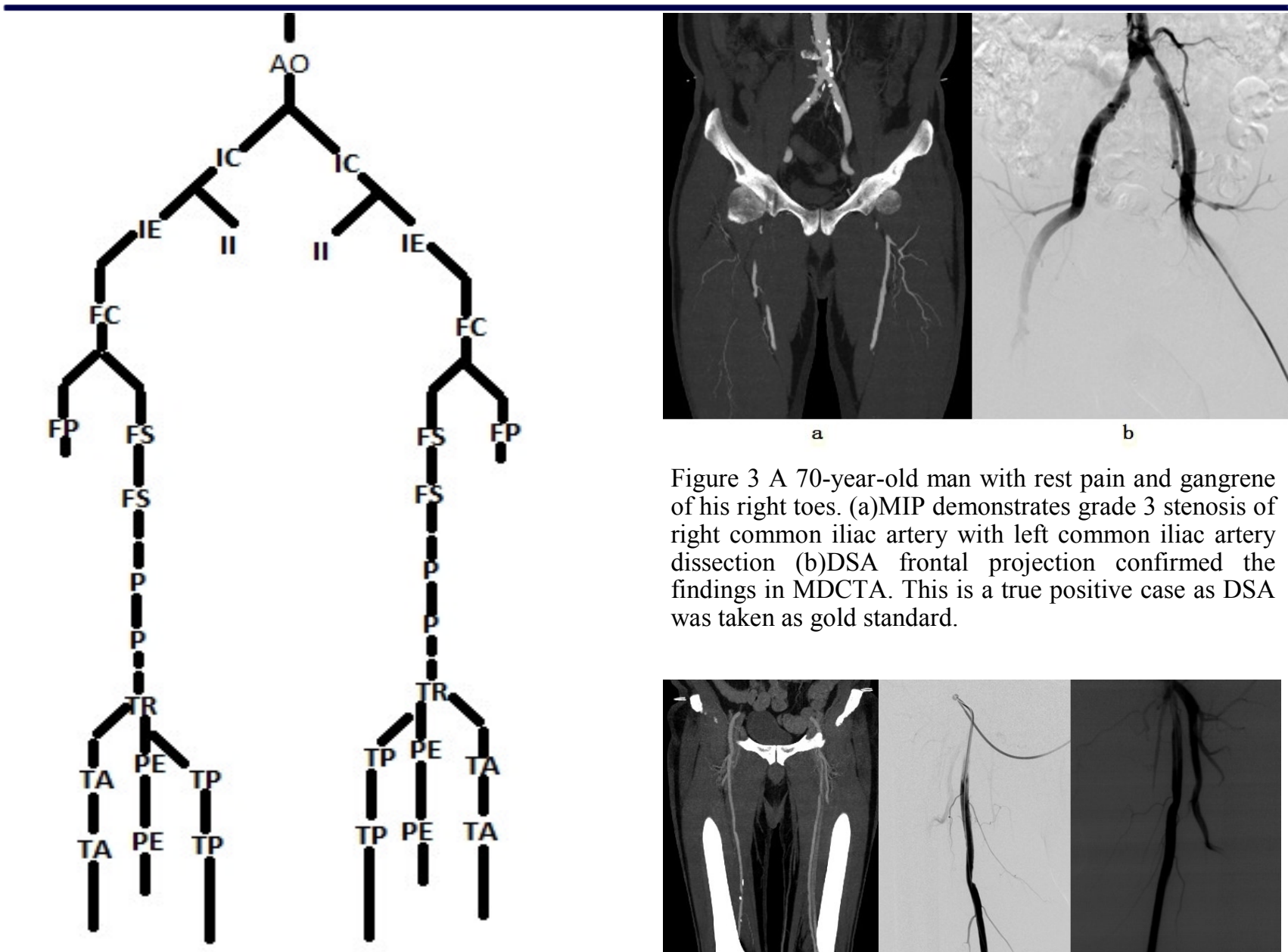

Figure 3 A 70-year-old man with rest pain and gangrene of his right toes. (a)MIP demonstrates grade 3 stenosis of right common iliac artery with left common iliac artery dissection (b)DSA frontal projection confirmed the findings in MDCTA. This is a true positive case as DSA was taken as gold standard.

Figure 1 Segmentation of the arterial tree of lower limb in our study. Infrarenal aorta (AO), common iliac (CI), internal iliac (II), external iliac (EI), common femoral (CF), deep femoral(DF), proximal (SF1) and distal superficial femoral (SF2), supra-articular (P1) and subarticular popliteal (P2), proximal(AT1) and distal (AT2) anterior tibial, tibioperoneal(TR), proximal (PE1) and distal peroneal (PE2), and proximal(TP1) and distal (TP2) posterior tibial.
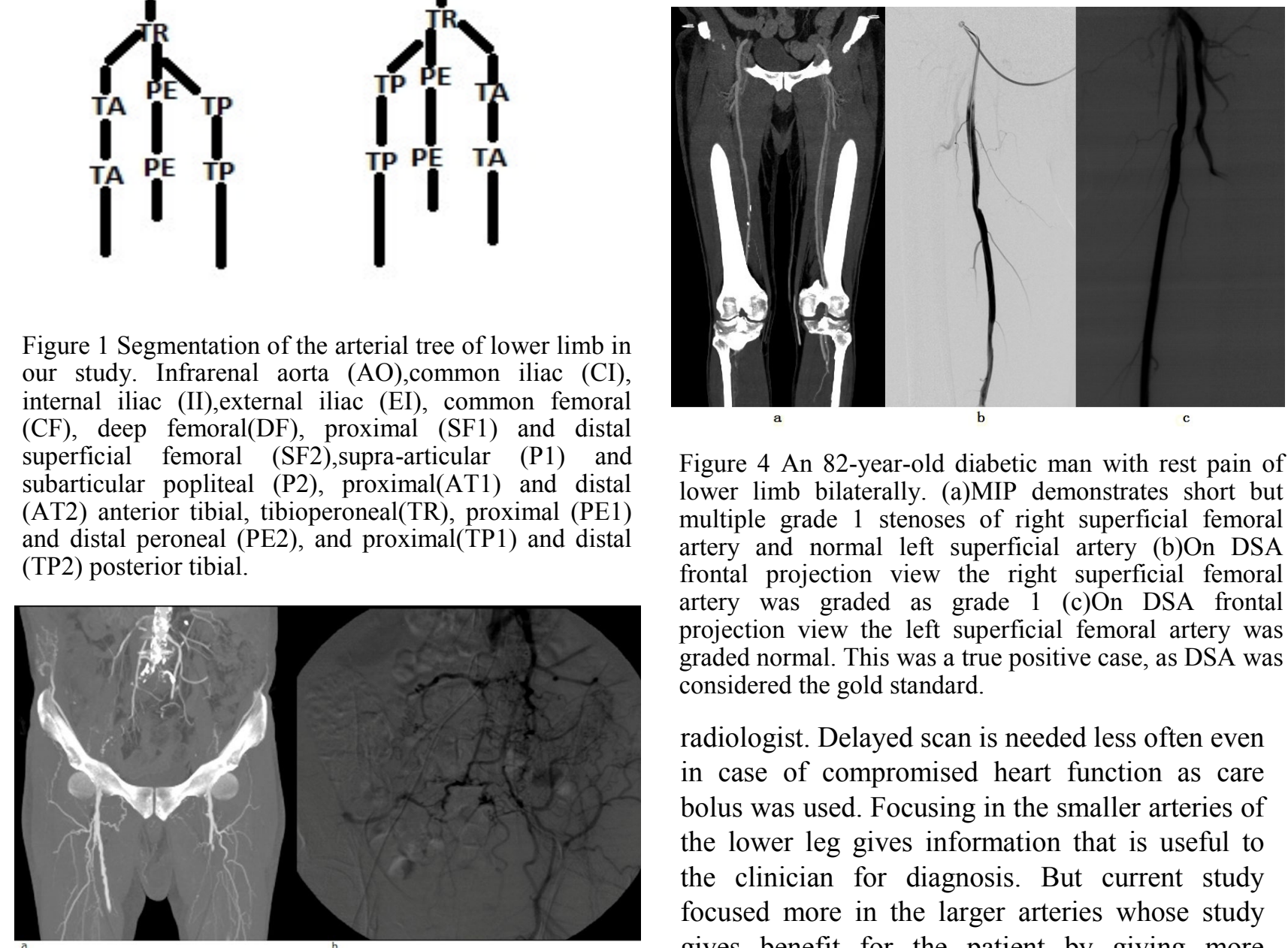

Figure 4 An 82-year-old diabetic man with rest pain of lower limb bilaterally. (a)MIP demonstrates short but multiple grade 1 stenoses of right superficial femoral artery and normal left superficial artery (b)On DSA frontal projection view the right superficial femoral artery was graded as grade 1 (c)On DSA frontal projection view the left superficial femoral artery was graded normal. This was a true positive case, as DSA was considered the gold standard.

radiologist. Delayed scan is needed less often even in case of compromised heart function as care bolus was used. Focusing in the smaller arteries of the lower leg gives information that is useful to the clinician for diagnosis. But current study focused more in the larger arteries whose study gives benefit for the patient by giving more practical information to the clinician and interventional radiologist. So we used delayed scanning technique less often. This could be the reason why the sensitivity and specificity in our study is slightly lower. Moreover, these values also bear excellent comparison with values of 92 
Table 5 Comparison of Studies Using MDCTA for the Detection of Stenosis

\begin{tabular}{|c|c|c|c|c|c|c|c|c|}
\hline & & & Aorto-il & & Femoro & pliteal & Infra & pliteal \\
\hline Author & $\begin{array}{l}\text { No. of } \\
\text { patients }\end{array}$ & Slices & Sens(\%) & $\operatorname{Spec}(\%)$ & Sens(\%) & $\operatorname{Spec}(\%)$ & $\begin{array}{l}\text { Sens } \\
(\%)\end{array}$ & $\operatorname{Spec}(\%)$ \\
\hline Ota et al. ${ }^{9}$ & 24 & 4 & 97 & 100 & 100 & 96 & 100 & 100 \\
\hline $\begin{array}{l}\text { Catalano et } \\
\text { al. }{ }^{22}\end{array}$ & 50 & 4 & 95 & 90 & 98 & 96 & 96 & 93 \\
\hline $\begin{array}{l}\text { Portugaller et } \\
\text { al. }^{23}\end{array}$ & 50 & 4 & 88 & 75 & 95 & 70 & 86 & 74 \\
\hline Laswed et al. ${ }^{10}$ & 34 & 16 & 95 & 100 & 95 & 95 & 91 & 96 \\
\hline $\begin{array}{l}\text { Willmann et } \\
\text { al. }{ }^{19}\end{array}$ & 39 & 16 & 99 & 98 & 98 & 96 & 97 & 96 \\
\hline $\begin{array}{l}\text { Schernthaner } \\
\text { et al. }{ }^{24}\end{array}$ & 50 & 16 & 100 & 100 & 97 & 99 & 98 & 100 \\
\hline Fine et al. ${ }^{12}$ & 107 & 64 & 86 & 95 & 90 & 90 & 90 & 96 \\
\hline $\begin{array}{l}\text { Shareghi et } \\
\text { al. }{ }^{25}\end{array}$ & 28 & 64 & 100 & 99 & 100 & 99 & 97 & 99 \\
\hline $\begin{array}{l}\text { N. Fotiadis et } \\
\text { al. }{ }^{15}\end{array}$ & 41 & 64 & 100 & 100 & 100 & 97 & 98 & 97 \\
\hline Current study & 42 & 128 & 84 & 93 & 86 & 94 & 96 & 86 \\
\hline
\end{tabular}

to $100 \%$ and 91 to $99 \%$, respectively, for contrastenhanced MR angiography. ${ }^{20}$ Segments that were not of diagnostic quality due to inadequate opacification, extreme calcification, or artifacts were excluded from the analysis.

Finally our study shows that for larger arteries which the clinician and interventional radiologist are more concerned, MDCTA appears consistent and accurate in the assessment of patients with peripheral occlusive disease of larger arteries like aorto-iliac and poplitiofemoral region and can be reliably used to grade disease severity and plan treatment while it is less consistent and less accurate for smaller arteries like infra-popliteal region.

It should also be borne in mind that whereas MDCTA is a 3D technique permitting image reconstruction in multiple planes, DSA is solely a 2D technique in which images are obtained in just two or three planes at best. But recent advances in DSA like rotational DSA which can produce 3D images can overcome limitations of projection images taken from few angles in our study. So MDCTA is better than 2D DSA in this aspect.

In the present study diagnosis and grading of stenoocclusive disease was performed with routine analysis of the axial images and thin coronal, sagittal, and oblique MIPs. Curved planar reconstructions were performed only when needed and volume-rendering images were not used for diagnosis. Evaluation of axial and cross-sectional images is critical in grading the severity of stenotic disease in patients with diabetes and chronic renal failure due to extensive calcifications, which can often make standard reconstructive views deceptive. ${ }^{7,11,21}$ Radiologists are familiar with cross-sectional images, and with appropriate window adjustment it is possible to evaluate the patency and degree of stenosis even in small calcified tibial vessels.

\section{Limitations}

There were several limitations of the present study. First, it is limited principally by its retrospective nature further prospective work is certainly warranted to confirm our findings. Second the DSA images were all acquired as part of a therapeutic intervention and only the clinical relevant segments were imaged. However, all the DSA images included in the analysis were of high quality and represent the true extent of the disease.

\section{CONCLUSION}

MDCTA is an excellent alternative in diagnosing lower extremity arterial occlusive diseases above the knee. DSA remains better on illustrating distal 
runoff vessels. Larger is the size of arteries better is the performance of MDCTA as compared to DSA.

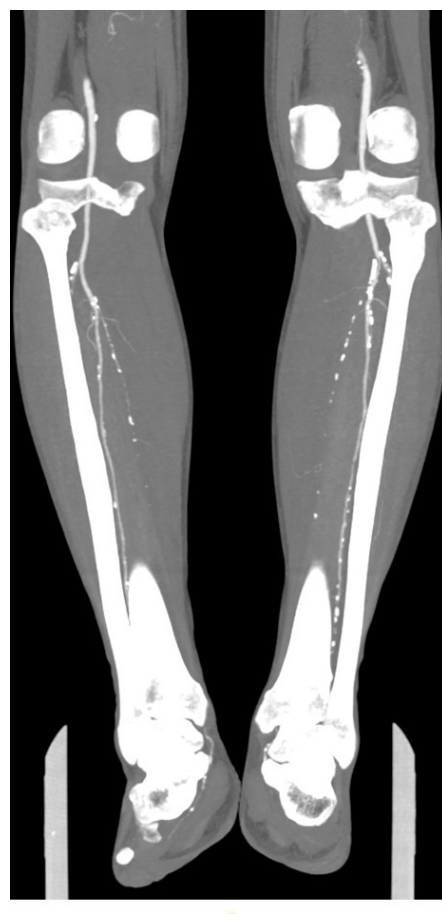

a

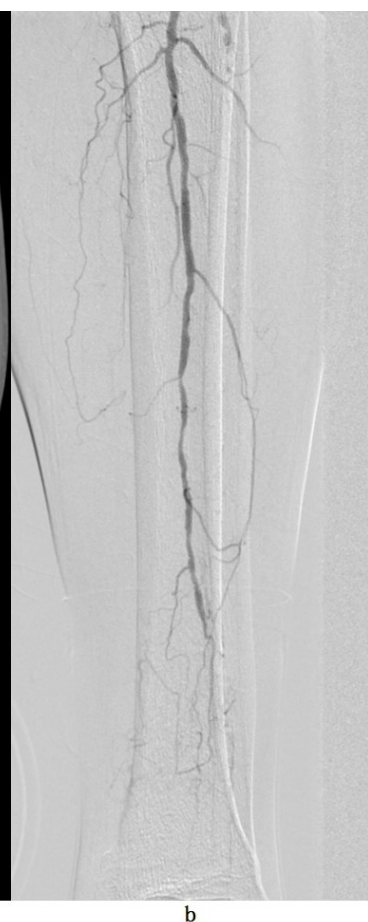

Figure 5 A 70-year-old diabetic man presented with left lower limb intermittent claudication. (a)MIP demonstrates calcification obscuring some portion of the left peroneal artery while the visible portion appears smooth and normal (b)On DSA frontal projection view the left peroneal artery was graded as grade 3 stenosis at multiple sites. This was a false positive case, as DSA was considered the gold standard

\section{REFERENCES}

1. Ricardo C, Moreira R. Surgical treatment of aorto-iliac occlusive disease. J Vasc Br. 2002;1:47-54.

2. NW S. Complications in peripheral vascular interventions: emerging role of direct thrombin inhibitors. J Vasc Interv Radiol. $2005 ; 16: 165-71$

DOI: 10.1097/01.RVI.0000147548.66405.84. PMID:15713917.

3. B.C. Meyer, A. Oildenburg, B.B. Frericks, C. Ribbe, W. Hopfenmuller, K.-J. Wolf, et al. Quantitative and qualitative evaluation of the influence of different table feed on visualization of peripheral arteries in CT angiography of aortoiliac and lower extremity arteries. Eur radiol 2008;18:1546-55. DOI: 10.1007/s00330-0080914-0. PMID:18379744.

4. Shareghi S, Gopal A, Gull K, Matchinson JC, Wong CB, Weinberg N, et al. Diagnostic Accuracy of 64 Multidetector Computed Tomographic Angiography in Peripheral Vascular Disease. Catheterization and Cardiovascular Interventions. 2010;75:23-31. PMID: 19753637

5. Alvarez SS, Mellado JM, Barea NY, Martin J, Garces RB, Alava SS. MDCT Angiography of the lower extremities: Utility and Limitations. RSNA; 2013; Chicago2013.

6. Sommer WH, Helck A, Bamberg F ea. Diagnostic value of time resolved CT angiography for the lower leg. EurRadiol 2010;20:2876-81. DOI: 10.1007/s00330-010-1861-0.

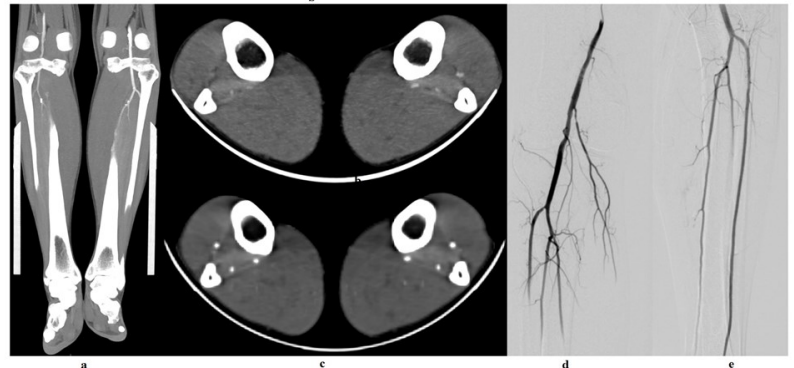

Figure 6 A 67-years-old man with bilateral intermittent claudication. (a)MIP does not demonstrate the branches below tibioperoneal trunk in regular scan (b) The crosssectional MIP shows better contrast enhancement of the run-off vessels in the left side (c)the cross-sectional MIP of the run-off vessels on delayed scan shows normal enhancement bilaterally .On DSA frontal projection view the run off vessels enhance normally bilaterally $(\mathrm{d}, \mathrm{e})$

7. Brockmann C, Jochum S, Sadick M ea. Dual-energy CT angiography in peripheral arterial occlusive disease. CardiovascInterventRadiol. 2009;32:630-7. DOI: $10.1007 /$ s00270-008-9491-5. PMID:19130122.

8. Willmann JK BB, Schertler T et al. Aortoiliac and lower extremity arteries assessed with 16-detector row CT angiography: prospective comparison with digital subtraction angiography. Radiology Sep. 2005;236:108393. DOI: 10.1148/radiol.2362040895. PMID:16055691.

9. D F, J. L. Peripheral CT angiography for interventional treatment planning. Eur Radiol. 2006;16:M58-M64. DOI: 10.1007/s10406-006-0197-y. PMID:18655268.

10. Z. S. Diagnostic accuracy of multislice CT angiography in peripheral arterial disease. J Vasc Interv Radiol 2006;17:1915-21.

DOI: 10.1097/01.RVI.0000248830.17550.50. PMID:17185686.

11. H O, K T, K I. MDCT compared with digital subtraction angiography for assessment of lower extremity arterial occlusive disease: importance of reviewing cross-sectional images. . AJR Am J Roentgenol Jan. 2004;182:201-9. DOI: 10.2214/ajr.182.1.1820201. PMID:14684540.

12. C C, F F, A L. Infrarenal aortic and lower-extremity arterial disease: diagnostic performance of multi-detector row CT angiography. Radiology May. 2004;231:555-63.

13. Portugaller HR, Schoellnast H, KA H. Multislice spiral CT angiography in peripheral arterial occlusive disease: a valuable tool in detecting significant arterial lumen narrowing? Eur Radiol Sep. 2004;14:1681-7. DOI: 10.1007/s00330-004-2289-1. PMID:15114490.

14. Laswed T RE, Guntern D, et al. Assessment of occlusive arterial disease of abdominal aorta and lower extremities arteries: value of multidetector CT angiography using an adaptive acquisition method. EurRadiol 2008;18:263-72. DOI: $10.1007 / \mathrm{s} 00330-007-0749-0$.

15. Schernthaner R SA, Lomoschitz F, et al. Multidetector CT angiography in the assessment of peripheral arterial occlusive disease: accuracy in detecting the severity, number and length of stenoses. EurRadiol. 2008;18:665-71. DOI: $10.1007 / \mathrm{s} 00330-007-0822-8$.

16. JJ F, PA H, JH R, LO B. 64-Slice peripheral computed tomography angiography: A clinical accuracy evaluation. $\mathrm{J}$ Am Coll Cardiol. 2006;47:1495-6. DOI: 10.1016/ j.jacc.2006.01.009. PMID:16580545.

17. Fotiadis N, Kyriakides C, Bent C, Vorvolakos T, Matson M. 64-section CT angiography in patients with critical limb ischaemia and severe claudication: Comparison with digital subtractive angiography Clin Radiol Oct. 2011;66:945-52. DOI: 10.1016/j.crad.2011.04.015. PMID:21658691. 
18. Met R, Bipat S, Legemate DA ea. Diagnostic performance of computed tomography angiography in peripheral arterial disease: a systematic review and meta-analysis JAMA. 2009;301:415-24.

19. Hellinger JC. Computed Tomographic Arteriography of the Upper and Lower Extremities. United Kingdom, London: Informa Healthcare; [Cited: Jan 2016]. Available from: www.informahealthcare.com.

20. Bezooijen R, van den Bosch HC, Tielbeek AV ea. Peripheral arterial disease: sensitivity-encoded multiposition MR angiography compared with intraarterial angiography and conventional multiposition MR angiography. Radiology. 2004;231:263-71. DOI: 10.1148/ radiol.2311020845. PMID:15068952.

21. BC M, T W, E F. Do the cardiovascular risk profile and the degree of arterial wall calcification influence the performance of MDCT angiography of lower extremity arteries? Eur Radiol 2010;20:497-505. DOI: 10.1007/ s00330-009-1555-7. PMID: 19789885. 